\title{
Psicoterapia de criança com alopecia areata universal: desenvolvendo a resiliência
}

\author{
Marina Menezes ${ }^{1}$ \\ Mariana López \\ Josiane da Silva Delvan \\ Universidade do Vale do Itajai, Itajai-SC, Brasil
}

\begin{abstract}
Resumo: Este artigo apresenta um estudo de caso de uma criança portadora de alopecia areata universal, vitiligo e transtorno de ansiedade generalizada, atendida em clínica-escola de psicologia de 2002 a 2007. A abordagem terapêutica adotada foi a psicoterapia de orientação psicanalítica infantil e a orientação sistemática aos pais, objetivando a diminuição dos sintomas e o desenvolvimento de habilidades para o enfrentamento da doença crônica. No decorrer do processo, ao longo dos cinco anos de atendimento, a paciente adaptou-se positivamente à doença, ocorrendo a remissão dos sintomas de ansiedade e o desenvolvimento de comportamentos resilientes que indicaram a alta terapêutica.
\end{abstract}

Palavras-chave: doenças crônicas, psicoterapia da criança, resiliência (psicologia).

\section{Psychotherapy of child with universal alopecia areata: developing resilience}

\begin{abstract}
This paper presents a study of case of a child with universal alopecia areata disease vitiligo, generalized anxiety disorder, taken care of in school-clinic of psychology in the period of 2002 to 2007. The therapeutical boarding was the psychotherapy of infantile psychoanalytical orientation and the systematic parent's orientation to reach the reduction of the symptoms and development of abilities for the confrontation of the chronic illness. During of the process, to the long of the five years of attendance, the patient presented positively adaptation to the illness, the remission of the anxiety symptoms and the development of resilient behaviors.
\end{abstract}

Keywords: chronic illness, child psychotherapy, resilience (psychological).

\section{Psicoterapia del niño con alopecia areata universal: desarrollando la resiliencia}

\begin{abstract}
Resumen: Este artículo presenta un estudio del caso de un niño con alopecia areata, vitíligo y trastorno de la ansiedad generalizada atendida en una clínica-escuela de psicología en el período del 2002 al 2007. El tratamiento terapéutico adoptado fue la psicoterapia de orientación psicoanalítica infantil y la orientación a los padres, buscando la reducción de los síntomas y el desarrollo de las capacidades para confrontación de la enfermedad crónica, ocurriendo la remisión de los síntomas de la ansiedad y el desarrollo de los comportamientos resilientes que habían indicado el fin terapéutico.
\end{abstract}

Palabras clave: enfermedades crónicas, psicoterapia infantil, resiliencia (psicologia).

A psicoterapia de orientação psicanalítica sustenta-se no pressuposto de que os conflitos internos inconscientes estão diretamente relacionados aos sintomas (Sours, 1996). Dessa forma, um distúrbio psicológico representa um obstáculo ao desenvolvimento emocional do indivíduo, que poderá caracterizar-se como imaturidade da capacidade de relacionar-se com as pessoas e com o ambiente. Assim, a psicoterapia objetiva desfazer esse obstáculo permitindo que o desenvolvimento "tenha lugar onde anteriormente não era possível” (Winnicott, 1986/1996, p. 81).

Uma das formas mais utilizadas para atingir a superação de conflitos das crianças é a psicoterapia lúdica e a orientação sistemática aos pais (Sours, 1996). A psicoterapia lúdica refere-se à utilização do brinquedo, durante o processo terapêutico, de uma forma simbólica que permita a criança a expressão, a comunicação, o acesso às fantasias, desejos, sentimentos e experiências em relação a si

\footnotetext{
${ }^{1}$ Endereço para correspondência:

Marina Menezes. Rua 1201, 120, apto 303. Centro. CEP: 88.330-792.

Balneário Camboriú-SC, Brasil. E -mail: mamenezes@terra.com.br
}

próprio e aos que fazem parte de seu convívio (Aberastury, 1982/1992; Aberastury, 1996; Glenn, 1996; Grünspun, 1997; Klein, 1955/1980). Na psicoterapia de orientação psicanalítica infantil, o conflito da criança é tratado a partir de interpretações, abrangendo a compreensão das fases do desenvolvimento (Sours, 1996). Porém, a técnica do brinquedo deve ser complementada com a fala do paciente, em especial no período de latência e puberdade, favorecendo o uso da linguagem verbal a fim de promover uma maior adaptação à realidade (Aberastury, 1996).

O psicoterapeuta de orientação psicanalítica infantil pode estimular a criança a desenvolver maneiras de modular suas exigências e/ou de utilizar defesas de modo adaptativo, buscando com isso que seus conflitos e os mecanismos de defesa se tornem conscientes, permitindo que as dificuldades emocionais sejam manejadas ou resolvidas por meio de progressivos insights (Sours, 1996).

No que se refere à orientação sistemática dos pais, Glenn, Sabot e Bernstein (1996), Grünspun (1997), Prado (1997) e Motta (2006) afirmam que orientar os pais da criança em processo psicoterapêutico pode auxiliar no esclarecimento da problemática infantil atual e às várias fases do desenvolvimento 
psíquico, discutindo as atitudes parentais diante do comportamento do filho, aumentando a eficácia do tratamento.

Ao favorecer o surgimento de comportamentos mais adaptados na criança através da psicoterapia e da orientação aos pais, o terapeuta auxilia o desenvolvimento de comportamentos resilientes na criança. A resiliência em psicologia refere-se aos processos que explicam a superação de crises e adversidades a que são expostos indivíduos, grupos ou organizações e que envolve a interação entre atributos individuais e recursos ambientais em um processo que resulta em superação e retorno ao padrão adaptativo inicial, sem que o indivíduo saia ileso desse processo (Klein \& Linhares, 2007).

As tarefas evolutivas da infância (desmame, retirada de fraldas, início da escolarização, entre outras) constituem-se em desafios que requerem o desenvolvimento de mecanismos de adaptação por parte das crianças. De modo geral, as tarefas evolutivas são vivenciadas pelas crianças de forma mais ou menos adaptada, pois os aspectos individuais, as relações familiares, o contexto onde a criança se encontra e as intercorrências durante a infância podem interferir nesse processo. A doença crônica infantil é considerada uma intercorrência que leva algumas crianças a enfrentar maiores dificuldades, podendo inclusive estar associada ao desenvolvimento e/ou surgimento de psicopatologias (Castro \& Moreno-Jiménez, 2007), pois, as doenças crônicas apresentam curso e duração longos, ocasionando algumas vezes a hospitalização, podendo ser incuráveis, capazes de deixar seqüelas e limitações às funções da pessoa, exigindo constantes adaptações ao meio (Barros, 2003; Castro \& Piccinini, 2002; Castro \& MorenoJiménez, 2007; Vieira \& Lima, 2002).

Para Castro e Piccinini (2002) a doença crônica pode afetar a dinâmica familiar e a criança de maneira mais ou menos negativa. Cada caso pode se diferenciar em virtude de inúmeros fatores relacionados à família e a criança. Nesse sentido, é possível compreender que muitas doenças podem provocar alterações físicas, emocionais e sociais, exigindo cuidados intensivos e conseqüentemente processos adaptativos. Assim, a relação entre resiliência e a situação de doença, é conforme Bianchini e Dell'Aglio (2006) contextualizada como a capacidade do indivíduo lidar com a doença e suas limitações, colaborando e aderindo ao tratamento, re-adaptando-se e sobrevivendo de forma positiva.

Em face ao exposto, o presente artigo tem como objetivo relatar e discutir a experiência clínica e o desenvolvimento de comportamentos resilientes de uma paciente infantil, portadora de doenças crônicas (alopecia areata universal e vitiligo), no decorrer dos cinco anos em que ocorreu seu processo psicoterapêutico em uma Clínica-Escola de Psicologia.

\section{Método}

\section{Participante}

Este estudo de caso refere-se ao processo psicoterapêutico de uma criança do sexo feminino, com diagnóstico de alopecia areata universal, vitiligo e transtorno de ansiedade generalizada.

\section{Procedimento de coleta de dados}

O atendimento iniciou-se no ano de 2002, quando a paciente estava com cinco anos de idade e encerrou-se em dezembro de 2007. Ao iniciar o processo psicoterapêutico na Clínica-Escola de Psicologia, os pais da criança assinaram um termo de consentimento em que se declaravam cientes e concordes quanto à utilização dos registros terapêuticos para fins científicos. Para a obtenção dos dados foram seguidos todos os cuidados éticos referentes ao sigilo e preservação da identidade dos sujeitos envolvidos, conforme o Código de Ética Profissional dos Psicólogos e a Resolução 196/1996 do Conselho Nacional de Saúde, de acordo com o Conselho Federal de Psicologia (CFP) 016/2000.

\section{Análise dos dados}

Os relatos dos atendimentos foram registrados na íntegra pelos acadêmicos/terapeutas, que, posteriormente, incluíram trechos das sessões e verbalizações da paciente nos relatórios elaborados ao final de cada ano de psicoterapia. O material clínico analisado foi constituído a partir da sistematização desses registros. O conteúdo das intervenções foi submetido a uma análise de conteúdo inspirada nos passos metodológicos descritos pela literatura disponível sobre abordagens qualitativas em pesquisa (Moraes, 1999). Para a análise dos dados procedeu-se à ordenação das falas, extraídas das transcrições. As sessões foram lidas exaustivamente, na seqüência cronológica em que ocorreram, o que possibilitou a organização e a sistematização das idéias centrais.

\section{Resultados e Discussão}

Os atendimentos à paciente foram individuais e semanais, sendo realizadas orientações mensais aos pais. $\mathrm{O}$ processo psicoterapêutico ocorreu durante o estágio curricular de psicologia clínica, sendo conduzido por cinco acadêmicos do último ano de graduação do Curso de Psicologia, que, sob supervisão, permaneceram cada um pelo período de um ano com a paciente. Vale ressaltar que os atendimentos realizados na Clínica-Escola estavam submetidos ao calendário escolar da universidade, ocorrendo a troca de acadêmico/terapeuta sempre que a graduação do mesmo era concluída.

\section{Avaliação psicológica}

A avaliação constituiu-se o primeiro passo antes do início da psicoterapia, incluindo as entrevistas diagnósticas com os pais e com a criança. A paciente em questão foi encaminhada à avaliação psicológica por sua médica dermatologista, em virtude de um quadro crônico de perda total de pêlos e cabelos denominado alopecia areata universal, que pode ser desenvolvida em qualquer faixa etária e em ambos os sexos. A alopecia caracteriza-se como uma doença auto-imune e genética, cuja etiologia é provavelmente multifatorial, sendo 
ainda possivelmente influenciada por traumas psíquicos ( $\mathrm{Ri}$ vitti, 2005).

A paciente, filha única de um jovem casal, apresentou a primeira queda de cabelos aos oito meses de idade, período em que seus pais vivenciaram uma crise conjugal que culminou em uma separação temporária do casal, sendo que neste período, a mãe apresentou sintomas depressivos. A esse respeito, Sacristán, Oyola e Andaluz (2000) indicam que a presença de separações, maus tratos e transtornos mentais nos genitores está associada com uma probabilidade maior da criança apresentar dificuldades psicológicas na infância e adolescência.

O casal logo voltou a conviver e mudou-se de cidade. Neste período a criança apresentou repilamento parcial, sem longa duração, ocorrendo a perda de cabelos posteriormente. Os episódios de repilamento e queda dos cabelos passaram a ser uma constante na vida da paciente, suscitando expectativas acerca do crescimento total dos cabelos que nunca se concretizaram. Em especial na infância e na adolescência, a alopecia pode ocasionar sentimentos de baixa auto-estima, insegurança e dependência a um adulto, bem como transtornos psicológicos, em virtude de estar relacionada a períodos de desenvolvimento da identidade (Bellet \& Prose, 2005; Papadopoulos, Schwartz, \& Janniger, 2000; Rivitti, 2005).

A paciente apresentava também manchas brancas pelo corpo (rosto, braços e abdômen), denominadas de vitiligo, uma doença idiopática, cutânea e adquirida, caracterizada pelo aparecimento de nítidas manchas despigmentadas, localizadas em qualquer região da pele (Bellet \& Prose, 2005; Steiner, Bedin, Moraes, Villas, \& Steiner, 2004). Geralmente o vitiligo ocorre em associação com doenças auto-imunes, tais como: tireoidites, diabetes mellitus e alopecia areata (Bellet \& Prose, 2005; Steiner e cols., 2004).

Através das entrevistas de avaliação, observou-se que o comportamento dos pais frente ao diagnóstico médico era de superproteção e culpa pela dificuldade em lidar com a doença da filha. Segundo os pais, a despigmentação da pele em algumas regiões do corpo da paciente acentuava-se em períodos de ansiedade e estresse elevado, como apresentações e festas escolares.

A paciente queixava-se ainda de sintomas somáticos, como dores abdominais e cefaléia, sendo que o conjunto desses sintomas forneceu indicações para o diagnóstico de transtorno de ansiedade generalizada, que inclui o transtorno de excesso de ansiedade da infância (American Psychiatric Association [APA], 2002). A partir de tal diagnóstico, realizado pelo médico neurologista da paciente, a mesma passou a fazer uso de fluoxetina. Para Sacristán (2000) as enfermidades e as dificuldades intrafamiliares estão entre os fatores precipitantes da ansiedade na infância, sendo que tais situações têm relação direta com o processo de desenvolvimento da criança. A esse respeito, Halpern e Figueiras (2004) indicam a importância dos profissionais da saúde identificarem precocemente os fatores individuais e ambientais que podem representar fatores de risco a saúde mental infantil.

\section{Primeiro ano de psicoterapia}

Depois de realizada a avaliação, deu-se inicio ao processo de psicoterapia e a abordagem utilizada foi a psicoterapia de orientação psicanalítica infantil, que segundo Kernberg (1999) possui os seguintes objetivos: (a) a resolução de conflitos a partir do nível de desenvolvimento da criança; (b) a expressão de sentimentos; (c) a estimulação da retomada do desenvolvimento normal da criança; (d) a redução da ansiedade; (e) melhora da auto-estima; (f) melhora da tolerância à frustração; $(\mathrm{g})$ desaparecimento de sintomas; (h) criação de estratégias para lidar com situações de crise; (i) desenvolvimento da independência apropriada; (j) ampliação da boa relação com crianças e adultos; (k) manutenção de rendimento escolar satisfatório; e (1) favorecimento de insight visando a tomada de consciência das motivações pessoais e sociais para os comportamentos, principalmente em relação aos conflitos atuais da paciente. Tais objetivos nortearam o trabalho psicoterapêutico adotado e para tanto, foi utilizado o brinquedo durante as sessões de psicoterapia, considerando que o mesmo permite o acesso aos conflitos intrapsíquicos e à vida de relação da criança (Grünspun, 1997).

Durante o primeiro ano de psicoterapia, os pais da paciente identificaram sua dificuldade em aceitar a ausência de cabelos da filha como algo que não favorecia a convivência com crianças da mesma idade fora do âmbito da escola. Neste período do tratamento, a paciente usava sempre chapéus ou toucas que, segundo a mesma, protegiam-na dos olhares que a ausência de cabelos provocava nas pessoas, pois relatava: sempre me perguntam se tenho câncer ou aids e isso me incomoda e dá raiva (sic). Não demonstrava interesse em desenhar ou utilizar outra forma lúdica que representasse a ausência de cabelos e quando verbalizava sobre este tema, mostrava-se irritada e evitava entrar em contato com o mesmo, evidenciando defesas frente à ansiedade que este assunto suscitava.

Segundo Reiter-Purtill e Noll (2003) as doenças crônicas são consideradas estressores em potencial, sendo que em muitos casos, as condições crônicas ou os tratamentos causam restrições físicas e afetam as atividades diárias das crianças. Além disso, podem provocar mudanças na aparência física, gerando autopercepção negativa e reações negativas entre os colegas, pois a aparência é considerada um poderoso preditor de amizade e aceitação entre os pares. Dessa forma, neste primeiro ano de psicoterapia, foram realizadas intervenções que objetivavam estimular na criança e nos pais um possível fortalecimento da auto-estima e aceitação da aparência física, a fim de desenvolver comportamentos mais adaptativos diante dos aspectos físicos e sociais relacionados à doença. Tais intervenções representam procedimentos técnicos que fazem parte da perspectiva da psicoterapia de orientação psicanalítica, visto que esta preconiza que os procedimentos adotados pelo terapeuta devem sempre ser avaliados e validados em função dos objetivos propostos em psicoterapia (Cassorla, 2003). 
Castro (2007) ressalta que as conseqüências psicológicas de uma doença crônica e seu tratamento podem ser variadas e com efeitos a curto e longo prazo, tanto para a criança, quanto para sua família, assim, a intervenção deve focar o paciente e o grupo familiar. Essa foi a postura adotada para os pais da paciente, que compareciam regularmente às orientações, sendo que os temas abordados nestes encontros se referiam à importância do desenvolvimento da autonomia da criança e ao enfrentamento da doença crônica e da ansiedade.

\section{Segundo ano de psicoterapia}

No segundo ano de psicoterapia ocorreu mudança do acadêmico/terapeuta e da sala de atendimento, o que causou uma ruptura na aliança de tratamento. Para Sandler (1990) a forma como a criança lida com esta situação poderá demonstrar seu grau de maturidade e as defesas de que dispõe para manejar tais mudanças, pois, uma reação madura ou mais adaptativa é observada quando a criança é capaz de diferenciar entre a função do terapeuta como pessoa real e sua função como profissional. Para tanto, fatores como a idade da criança e predomínio de determinadas defesas devem ser considerados, pois a mudança de terapeuta poderá ainda estimular a transferência e a criação da resistência. Isto foi observado na paciente durante o estabelecimento do vínculo com o novo acadêmico/terapeuta, pois, comparava-o constantemente com o anterior, indicando que as mudanças, tanto de terapeuta quanto de sala de atendimento, representavam experiências dolorosas.

Quando foi formado um vínculo satisfatório com o novo acadêmico/terapeuta, este introduziu o tema: presença e ausência de cabelos por meio de livros infantis, figuras e recortes de revistas, que foram disponibilizados à paciente. Para Cassorla (2003) na psicoterapia de orientação psicanalítica, visa-se a ampliação da capacidade de pensar do paciente, que ocorre mediante o fortalecimento das suas funções egóicas e das maneiras de lidar com a realidade, o que justifica a utilização de procedimentos como orientação, cuidados, sugestões e uso de tratamentos biológicos. Nesse sentido, os procedimentos são sempre determinados pela necessidade de auxiliar o paciente a lidar melhor com os aspectos da realidade, o que foi considerado determinante para a paciente em questão. Quando o tema: cabelos surgia nas sessões, tal como no ano anterior, a paciente demonstrava irritação, porém, também passou a expressar raiva e a esperança de que os cabelos crescessem. Estes sentimentos passaram a ser simbolizados por ela através de músicas e desenhos, indicando um maior incremento na capacidade de representação do conflito.

\section{Terceiro ano de psicoterapia}

A continuação do tratamento da paciente alcançou seu terceiro ano com os pais relatando em uma das orientações que a filha ainda estava muito nervosa, ansiosa e que não apresentava tolerância à frustrações (sic). $\mathrm{O}$ vínculo com o terceiro acadêmico/terapeuta deu-se de forma mais adaptada do que no ano anterior, pois, ao contrário do que ocorreu na primeira troca de terapeutas, foi possível estabelecer um período de transição entre o acadêmico/terapeuta anterior e o atual, permitindo que ao término do segundo ano, a paciente já tivesse realizado contato com o terceiro acadêmico/terapeuta. Para Lhullier, Nunes, Antochevis, Porto e Figueiredo (2000) as mudanças de terapeuta em instituições de treinamento são muito comuns, visto que nem todos os tratamentos são concluídos durante o estágio do terapeuta em formação. Em função disso, a realização de um período de transição ou co-terapia como forma de adaptação à mudança pode resultar em um recurso importante para a não desistência do tratamento, favorecendo a adesão ao mesmo. De forma geral, a paciente demonstrou aceitar as atividades que incluíam desenhos sobre seus sentimentos e o uso de figuras para a composição de histórias propostas pelo novo acadêmico/terapeuta. Em uma das sessões relatou estar pronta para conversar sobre cabelos e sobre o impacto que a ausência dos mesmos causava em sua vida. Neste mesmo ano observou-se a diminuição dos comportamentos de superproteção dos pais e maior aceitação da condição da filha.

\section{Quarto ano de psicoterapia}

No quarto ano de tratamento, a paciente verbalizou em uma das sessões estar acostumada com a mudança de acadêmico/terapeuta, que também ocorreu mediante um período de transição. Nesta fase da psicoterapia, a criança passou a expressar seus sentimentos e pensamentos em relação à possibilidade de utilizar perucas no lugar de chapéus ou toucas, indicando menor presença de comportamentos ansiosos e maior flexibilidade para mudanças. A medicação foi então suspensa por ordem médica, em função da remissão dos sintomas de ansiedade.

\section{Quinto ano de psicoterapia}

Durante o ano de 2007, após um curto período de coterapia, ocorreu nova troca de acadêmico/terapeuta. A paciente, bem como seus pais, demonstraram comportamentos mais adaptativos em relação à doença. Neste período a criança relatou em uma das sessões: antes não conseguia sair sem meus pais e agora adoro sair sozinha. Através de alguns insights, revelou em uma outra sessão de atendimento que se sentia melhor em relação a não ter cabelos e à vergonha de ser diferente. Para Sandler (1990) no insight, o nível conceitual e intelectual da criança afeta sua visão de mundo e de si mesma perante seus sentimentos, além disso, a auto-observação pode auxiliar na superação do conflito interno (Sandler, 1990). A paciente demonstrou ter capacidade para lidar com estas adversidades durante o processo de psicoterapia, o que sugere o desenvolvimento de características resilientes. Acredita-se que a paciente já possuísse tais características e que através da psicoterapia, as mesmas puderam emergir. Nesse sentido, sugere-se ainda que, além do amadurecimento decorrente do próprio processo de desenvolvimento, cujo curso mostrava-se ocorrendo de forma plena, a criança 
beneficiou-se do processo psicoterapêutico, mesmo com as constantes trocas de acadêmico/terapeutas, pois as habilidades para enfrentar suas doenças crônicas, o reconhecimento de suas limitações, a adesão ao tratamento, assim como o suporte social e familiar, entendidos aqui como características resilientes, puderam ser estimuladas e desenvolvidas, permitindo a sua adaptação de forma positiva.

Para Castro e Moreno-Jiménez (2007) existem pessoas que podem possuir características resilientes transitórias e especificas de alguns contextos. A resiliência não é um atributo fixo do indivíduo, pois a pessoa pode superar um obstáculo em determinado momento, contudo, pode não se adaptar tão bem em outro momento da vida (Yunes, 2003).

No ano de 2007 durante as orientações, os pais da paciente relataram sentirem-se mais seguros no manejo da filha, desejando ampliar a família, o que se concretizou com a gravidez da mãe. Assim a paciente viu-se ocupando um novo papel, o de irmã mais velha, até então desconhecido e de modo geral, durante este ano, demonstrou habilidade para superar novos e velhos desafios, reconhecendo suas limitações de forma adaptativa, verbalizando em um dos atendimentos que talvez utilizasse perucas quando estivesse na adolescência.

A adaptação positiva na doença crônica também está associada à presença de fatores que de acordo com Castro e Moreno-Jiménez (2007) podem ser compreendidos como: (a) Fatores de risco: variedade da doença (gravidade, visibilidade, problemas médicos associados); nível de funcionamento que não depende da criança; e estressores psicossociais que se relacionam com a doença; (b) Fatores de proteção: variáveis intrapessoais (temperamento, habilidade para resolver problemas); ambiente familiar; apoio social; recursos da comunidade; habilidades no processamento do estresse (estratégias de elaboração de enfrentamento). Wallander, Thompson Jr. e Alriksson-Schimidt (2003) indicam que o funcionamento e o ajustamento familiar ao estresse, assim como relacionamento da criança e seus pares também fazem parte dos aspectos relacionados ao ajustamento positivo das crianças que apresentam condições crônicas de saúde.

$\mathrm{Na}$ paciente em questão, os fatores de proteção foram incrementados e ampliados com apoio da psicoterapia e da orientação aos pais. Pesce, Assis, Avanci, Santos, Malaquias e Carvalhaes (2005) sugerem três fatores que estão relacionados à proteção em crianças diante situações adversas, sendo estes identificados como características que foram focadas no processo psicoterapêutico desta paciente: (a) desenvolvimento de características de personalidade como a auto-estima, a flexibilidade e a habilidade para resolução de conflitos; (b) estímulo à coesão e bom relacionamento familiar; e (c) disponibilidade de suporte externo a fim de encorajar e reforçar as estratégias de enfrentamento.

Meneses e Ribeiro (2000) ressaltam que a psicoterapia, a informação sobre a doença e a promoção da saúde, são essenciais para desenvolver auto-estima e afeto positivo. Outras estratégias também auxiliam no enfrentamento da doença crônica na infância, entre elas estão: o brinquedo e a diversão, as atividades lúdicas; a presença de pais, familiares e amigos durante o tratamento e a escolarização (Castro \& Piccinini, 2002; Vieira \& Lima, 2002).

A adaptação às mudanças apresentadas pela paciente destacou a proximidade da alta terapêutica, pois segundo o relato da criança em uma das sessões do ano de 2007: a terapia ajudou-me a mudar bastante, fez parte da minha vida; sei que logo não vou mais precisar vir aqui. Diante do término do tratamento, é comum que o paciente anuncie que deixará a terapia, pois percebe a possibilidade de alta. Entretanto para que esta seja efetivada, é essencial uma decisão conjunta entre os interessados e tempo hábil para trabalhar a separação. O final do tratamento deve ser gradual, pois o paciente pode vivenciar sentimentos de dor, perda e luto, assim como conquistas, expectativas e alegrias pela tarefa ter sido concluída (Castro, 1989).

No final do ano de 2007, em uma das últimas sessões, a paciente expressou simbolicamente através de um desenho, os cinco anos de psicoterapia, bem como os cincos acadêmicos/terapeutas que a atenderam, verbalizando que conseguia observar como sua história havia se modificado, conforme destaca o relato abaixo:

No primeiro ano eu era super nervosa e medrosa... eu tinha vergonha até das pessoas... no segundo $e$ no terceiro ano eu comecei a falar mais dos meus sentimentos e comecei a aprender a lidar com meus problemas. No quarto ano eu acho que já comecei a superar meus problemas, eu me sentia bem melhor! E este ano eu me sinto super bem, me sinto calma e sinto que agora superei meus problemas.

Castro (1989) aponta que quando a alta está próxima, a criança é capaz de fazer relatos diretos de suas atividades do dia-a-dia, com maior expressão verbal para comunicar sentimentos e desejos, além de maior capacidade de sublimar e enfrentar a realidade.

Assim, após cinco anos de tratamento psicoterapêutico, em dezembro de 2007 encerrou-se o atendimento da paciente, que durante tal processo, demonstrou o desenvolvimento de comportamentos resilientes que potencialmente estavam presentes em si e que foram incrementados a partir da psicoterapia, possibilitando maior nível de adaptação positiva à realidade das suas doenças crônicas e dos efeitos que as mesmas provocavam em sua vida e na de sua família.

Sugere-se que este processo psicoterapêutico estimulou a manifestação de comportamentos resilientes, diminuindo as angústias e sintomas da paciente no enfrentamento da doença. A orientação aos pais também possibilitou modificações positivas na dinâmica familiar e na evolução da criança. Estes comportamentos adaptativos puderam nortear o processo de alta, porém, infere-se que mesmo com o término da psicoterapia, a criança em seu processo de desenvolvimento, maturação e expansão da personalidade, poderá deparar-se 
com novas tarefas evolutivas, seguidas de crises mais ou menos intensas, as quais terá que enfrentar apoiada em suas condições internas e externas.

\section{Considerações finais}

São consideradas crianças resilientes aquelas que passaram por sérios traumas e que apresentam uma diminuição de riscos ou a até mesmo a ausência do diagnóstico psicopatológico. Em outros casos, a criança resiliente é aquela que apresenta maturidade diante da situação conflitante e se adapta em sua vida cotidiana (Castro \& Moreno-Jiménez, 2007).

Para estimular a criança a desenvolver aspectos positivos de adaptação, ou seja, resiliência, sugere-se que o psicólogo clínico infantil reafirme para a criança através da psicoterapia, a importância de sua auto-estima, do respeito ao próprio corpo, informando seus direitos, reforçando o diálogo e a tolerância na família, possibilitando a prevenção de psicopatologias. Portanto, um dos objetivos da psicoterapia infantil com pacientes portadores de doença crônica, ancorase no auxílio para a superação das tarefas evolutivas, tendo em vista um desenvolvimento razoável dentro das limitações decorrentes de cada quadro clínico (Castro \& Moreno-Jiménez, 2007).

Para ampliar a possibilidade do paciente desenvolver comportamentos resilientes, o psicólogo pode ainda estimular pela via terapêutica, a habilidade da criança utilizar as situações adversas em seu processo de desenvolvimento pessoal e crescimento social, conforme o presente estudo de caso demonstrou. Tais estratégias permitem auxiliar a criança e sua família a enfrentar os problemas de forma positiva e criativa, o que não significa não estar mais em contato com os fatores de risco, mas desenvolver comportamentos de enfrentamento e engajamento para superar determinados desafios e problemas.

\section{Referências}

Aberastury, A. (1992). Psicanálise da criança: Teoria e técnica (8a ed.). Porto Alegre: Artes Médicas. (Original publicado em 1982)

Aberastury, A. (1996). Abordagens à psicanálise de crianças. Porto Alegre: Artes Médicas.

American Psychiatric Association. (2002). DSM-IV-TR: Diagnostical and statistical Manual of Mental Disorders (C. Dornelles, Trad., 4a ed.). Porto Alegre: Artmed.

Barros, L. (2003). Psicologia pediátrica: Perspectiva desenvolvimentista (2a ed.). Lisboa: Climepsi.

Bellet, J. S., \& Prose, N. S. (2005). Vitiligo em crianças: Uma revisão de classificação, hipóteses sobre patogênese e tratamento. Anais Brasileiros de Dermatologia, 80, 631- 636.

Bianchini, D. C. S., \& Dell'Aglio, D. D. (2006). Processos de resiliência no contexto de hospitalização: Um estudo de caso. Paidéia (Ribeirão Preto), 16, 427-436.
Cassorla, R. M. S. (2003). Procedimentos, colocação em cena da dupla ("enactment") e validação clínica em psicoterapia psicanalítica e psicanálise. Revista de Psiquiatria do Rio Grande do Sul, 25, 426-435.

Castro, E. K. de (2007). Psicologia pediátrica: A atenção à criança e ao adolescente com problemas de saúde. Psicologia: Ciência e Profissão, 27, 396-405.

Castro, E. K., \& Moreno-Jiménez, B. (2007). Resiliência en niños enfermos crónicos: Aspectos teóricos. Psicologia em Estudo, 12, 81-86.

Castro, E. K. de, \& Piccinini, C. A. (2002). Implicações da doença orgânica crônica na infância para as relações familiares: algumas questões teóricas. Psicologia: Reflexão e Critica, 15, 625-635.

Castro, M. da G. K. (1989). Término e critérios de alta em psicoterapia infantil. In I. Duarte, I. Bornholdt \& M. G. K. Castro (Orgs.), A prática da psicoterapia infantil (pp. 51-71). Porto Alegre: Artes Médicas.

Glenn, J. (1996). Princípios gerais da análise de crianças. In J. Glenn (Org.), Psicanálise e psicoterapia de crianças (pp. 23-43). Porto Alegre: Artes Médicas.

Glenn, J., Sabot, L. M., \& Bernstein, I. (1996). O papel dos pais na análise de crianças. In J. Glenn (Org.), Psicanálise e psicoterapia de crianças (pp. 239- 258). Porto Alegre: Artes Médicas.

Grünspun, H. (1997). Psicoterapia lúdica de grupo com crianças. São Paulo: Atheneu.

Halpern, R., \& Figueiras, A. C. M. (2004). Influências ambientais na saúde mental da criança. Jornal de Pediatria, 80(Supl. 2), S104-S110.

Kernberg, P. F. (1999). Psicoterapia individual. In H. Kaplan \& B. Sadock (Orgs.), Tratado de psiquiatria (6a ed., Vol. 3, pp. 2602-2616). Porto Alegre: Artes Médicas.

Klein, M. (1980). A técnica psicanalítica através do brinquedo. In M. Klein, P. Heimann \& R. M. MoneyKyrle (Orgs.), Novas tendências na psicanálise (2a ed., pp. 25-48). Rio de Janeiro: Guanabara/Koogan. (Original publicado em 1955)

Klein, V. C., \& Linhares, M. B. M. (2007). Temperamento, comportamento e experiência dolorosa na trajetória de desenvolvimento da criança. Paidéia (Ribeirão Preto), 17, 33-44.

Lhullier, A. C., Nunes, M. L. T., Antochevis, A. F., Porto, A. M., \& Figueiredo, D. (2000). Mudança de terapeuta e abandono da psicoterapia em uma clínica-escola. Aletheia, 11, 7-11.

Meneses, R. F., \& Ribeiro, J. P. (2000). Como ser saudável com uma doença crónica: Algumas palavras orientadoras da acção. Análise Psicológica, 18, 523-528.

Moraes, R. (1999). Análise de conteúdo. Educação, 22(37), 7-32.

Motta, I. F. da (2006). Orientação de pais: Novas perspectivas no desenvolvimento de crianças e adolescentes. São Paulo: Casa do Psicólogo. 
Papadopoulos, A J., Schwartz, R. A., \& Janniger, C. K. (2000). Alopecia areata: Pathogenesis, diagnosis and therapy. American Journal of Clinical Dermatology, 1, 101-105.

Pesce, R. P., Assis, S. G., Avanci, J. Q., Santos, N. C., Malaquias, J. V., \& Carvalhaes, R. (2005). Adaptação transcultural, confiabilidade e validade da escala de resiliência. Cadernos de Saúde Pública, 21, 436-448.

Prado, L. C. (1997). Combinações terapêuticas no atendimento de crianças e adolescentes. In N. Fichtner (Org.), Prevenção, diagnóstico e tratamento dos transtornos mentais da infância e da adolescência: Um enfoque desenvolvimental (pp. 248-262). Porto Alegre: Artes Médicas.

Reiter-Purtill, J., \& Noll, R. B. (2003). Peer relationships of children with chronic illness. In M. C. Roberts (Ed.), Handbook of pediatric psychology (3a ed., pp. 176-197). New York: Guilford Press.

Rivitti, E. A. (2005). Alopecia areata: Revisão e atualização. Anais Brasileiros de Dermatologia, 80, 57-68.

Sacristán, J. R. (2000). La ansiedad y sus transtornos en la infancia. In J. R. Sacristán (Ed.), Psicopatología infantil básica (pp. 183- 194). Madrid: Pirámide.

Sacristán, J. R., Oyola, J. F. L., \& Andaluz, R. C. (2000). El desarrollo psicológico normal y patológico en la infancia: Los problemas psicopatológicos mayores y menores y sus causas. In J. R. Sacristán (Ed.), Psicopatología infantil básica (pp. 21-44). Madrid: Pirámide.

Sandler, J. (1990). Técnica da psicanálise infantil (2a ed.). Porto Alegre: Artes Médicas.

Sours, J. A. (1996). A aplicação de princípios da análise de crianças a formas de psicoterapia infantil. In J. Glenn (Org.), Psicanálise e psicoterapia de crianças (pp. 373390). Porto Alegre: Artes Médicas.

Steiner, D., Bedin, V., Moraes, M. B., Villas, R. T., \& Steiner, T. (2004). Vitiligo. Anais Brasileiros de Dermatologia, 79, 335-351.

Vieira, M. A., \& Lima, R. A. G. de. (2002). Crianças e adolescentes com doença crônica: Convivendo com mudanças. Revista Latino-Americana de Enfermagem, 10, 552-560.

Wallander, J. L., Thompson Jr., R. J., \& Alriksson-Schmidt, A. (2003). Psychosocial adjustment of children with chronic physical conditions. In M. C. Roberts (Ed.), Handbook of pediatric psychology (3a. ed., pp. 141-158). New York: Guilford Press.

Winnicott, D. W. (1996). Tudo começa em casa (2a ed.). São Paulo: Martins Fontes. (Original publicado em 1986)

Yunes, M. A. M. (2003). Psicologia positiva e resiliência: O foco no indivíduo e na família. Psicologia em Estudo (Maringá), 8(n.esp), 75-84.
Marina Menezes é Professora da Faculdade de Psicologia da Universidade do Vale do Itajaí, Centro de Ciências da Saúde, campus Itajaí.

Mariana López é mestranda em Psicologia pelo Programa de Pós-graduação em Psicologia da Universidade Federal de Santa Catarina, bolsista CAPES.

Josiane da Silva Delvan é Professora Doutora do curso de Psicologia da Universidade do Vale do Itajaí, Centro de Ciências da Saúde, campus Itajaí.
Recebido: 06/05/2008

$1^{a}$ revisão: 08/01/2009

$2^{a}$ revisão: 19/03/2009

Aceite final: 23/04/2009 